\title{
Parental influences run deep
}

The preferential expression of alleles that are inherited from one parent is known as genomic imprinting. This form of epigenetic regulation has been suggested to have a substantial role in brain development and function. However, the number of genes that show imprinting, as well as the anatomical location and developmental stage in which the expression bias occurs, remain unknown. Now, two studies by Gregg et al. provide evidence of widespread genomic imprinting

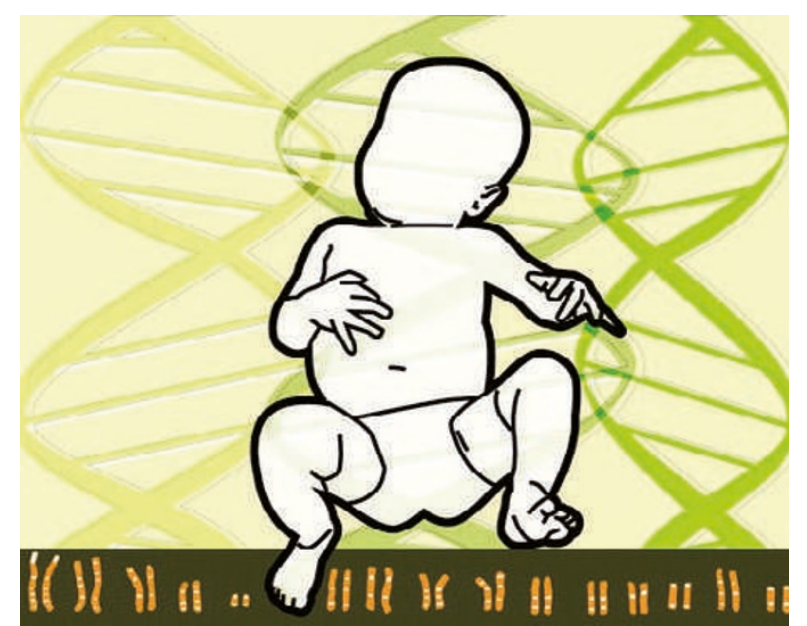

in the developing and adult mouse brain with complex patterns of spatial and temporal regulation.

The authors performed a genomewide analysis of RNA expression in the brain of mice at embryonic day 15 and in adults of both sexes. They identified over 1,300 gene loci that exhibited parental bias in the expression of a particular gene or specific transcript isoform. The parent-specific bias varied according to the animal's developmental stage: a predominantly maternal contribution was detectable in embryonic brains, whereas paternal alleles were preferentially expressed in adults. Moreover, the expression bias of imprinted genes was mainly detected in the hypothalamus and the prefrontal cortex - neural systems that are associated with feeding and motivated behaviours.

The sex of the animal was irrelevant for the expression of the majority of genes that exhibited a parental allelic effect. However, in the cortex, female mice preferentially expressed maternal alleles of genes that were located on the X chromosome. Females also had a much higher number of imprinted genes that were derived from autosomes, but in this case the bias was mainly paternal and was restricted to the hypothalamus. These findings reveal a novel mechanism for sexual dimorphism in the CNS.

The results indicate that biased expression of parental alleles may be a mechanism for the epigenetic regulation of brain function, with important implications for our understanding of neurological diseases. The studies reveal a very complex pattern of expression bias that is simultaneously dependent on the brain region, the developmental stage and the gene isoform. Future studies might elucidate the rules that govern genomic imprinting in the brain.

Cristian Bodo

ORIGINAL RESEARCH PAPERS Gregg, C. et al. High-resolution analysis of parent-of-origin allelic expresion in the mouse brain. Science 8 Jul 2010 (doi:10.1126/science.1190830) | Gregg, C. et al. Sex-specific parent-of-origin allelic expression in the mouse brain. Science 8 Jul 2010 (doi:10.1126/science.1190831)

FURTHER READING Wilkinson, L. S., Davies W. \& Isles A. R. Genomic imprinting effects on brain development and function. Nature Rev. Neurosci. 8, 832-843 (2007) | Jazin, E. \& Larry Cahill, L. Sex differences in molecular neuroscience: from fruit flies to humans. Nature Rev. Neurosci. 11, 9-17 (2010) 